\title{
MICROMACHINED THERMALLY ISOLATED CIRCUITS
}

\author{
Erno H. Klaassen", Richard J. Reay ${ }^{* *}$, Christopher Storment ${ }^{*}$, Jonathan Audy ${ }^{* * *}$, Peter Henry ${ }^{* * *}$, \\ A. Paul Brokaw ${ }^{* * *}$, and Gregory T.A. Kovacs \\ * Center for Integrated Systems, CIS-X 202, Stanford University, Stanford, CA 94305-4075 \\ ${ }^{* *}$ IBM Almaden Research Center, San Jose, CA 95120 \\ *** Analog Devices Inc., Santa Clara, CA 95052
}

\begin{abstract}
This paper details a post-process technique by which circuitry in an unmodified IC technology is thermally and electrically isolated from the silicon substrate. This method enables new applications for micromachining, including temperature regulation of analog integrated circuits. The process will be discussed in detail, along with improved tetramethyl ammonium hydroxide (TMAH) etching chemistries that use strong oxidizers to eliminate hillock formation. Also presented is an electrochemical biasing method that uses circuitry on the silicon being etched during the micromachining step. Work is currently underway to evaluate the use of these micromachining techniques for commercial analog circuit applications, several of which will be highlighted in this presentation.
\end{abstract}

\section{INTRODUCTION}

Silicon has a thermal conductivity close to that of aluminum $(1.57 \mathrm{~W} / \mathrm{cm} \cdot \mathrm{K}$ and $2.36 \mathrm{~W} / \mathrm{cm} \cdot \mathrm{K}$, respectively). This material property is highly beneficial for most circuits, since it allows heat generated in devices to be efficiently conducted into the package. There are applications, however, in which it is necessary to generate or detect precise, localized changes in temperature on the surface of a silicon chip. In order to do this without dissipating large amounts of power, the thermally conductive path through the silicon must be selectively removed so that peripheral circuitry still benefits from efficient cooling.

Techniques referred to as CMOS post-processing have been used to isolate regions of dielectric, inside which the interconnect layers in the process, usually aluminum and polysilicon, can be thermally isolated to form heating resistors and thermopiles. These devices can be processed using a front-side anisotropic undercut etch to create cantilevers [1] or a backside etch to form diaphragms [2].

A much wider range of applications is made possible with a new method that allows active devices to be thermally isolated. This is achieved by suspending silicon at the end of dielectric support beams. Entire circuits, or critical portions of circuits, can now be thermally isolated. These circuits can contain, depending on the IC process used, MOS and bipolar transistors, diffused resistors, pn diodes, and polysilicon resistors and capacitors. Thermally isolated circuits can be used for a wide range of applications involving temperature measurement of these suspended devices and low-power temperature regulation of thermally isolated circuitry.

\section{CIRCUIT ISOLATION PROCESS}

Figure 1 shows the post-processing fabrication method. Portions of the silicon substrate are exposed by superimposing the oxide cuts in the process [3]. Each oxide layer that is grown or deposited is removed in a subsequent etching step in these "open" regions. One or more n-wells are located at the ends of support beams defined by the exposed regions, as shown in Figure 1(a).

The wells are biased in tetramethyl ammonium hydroxide, an anisotropic silicon etchant, at $-0.8 \mathrm{~V}$ relative to an $\mathrm{Ag} / \mathrm{AgCl}$ electrode using a potentiostat. The p-type substrate is maintained near the open circuit potential at $-1.5 \mathrm{~V}$. A platinum counter electrode is used in this four-electrode potentiostat configuration [4]. The n-wells are passivated, while the p-substrate etches away in the typical anisotropic fashion. The substrate underneath the well is etched primarily along (I10) crystal planes.
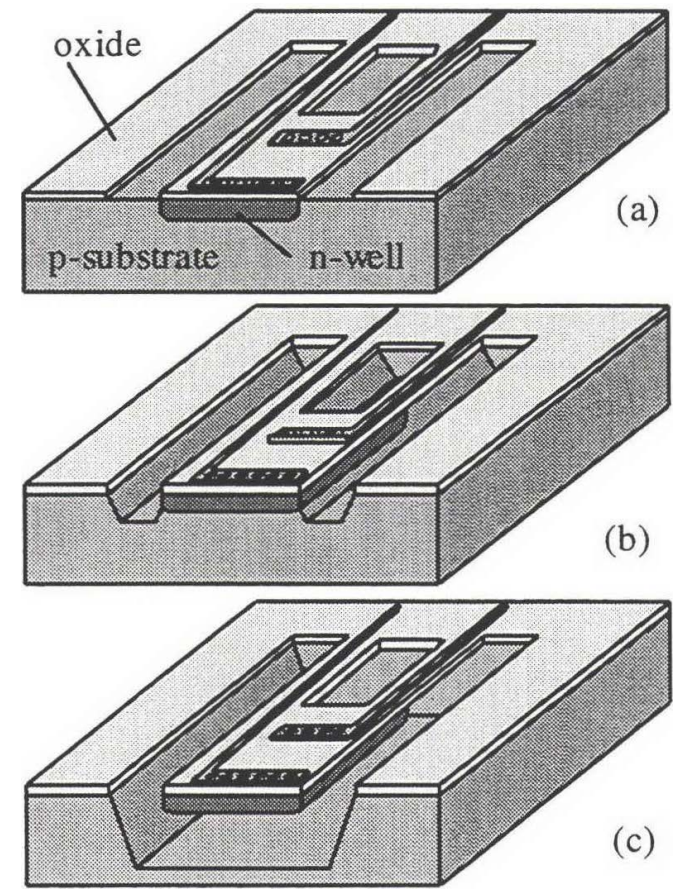

Figure 1. Circuit undercutting process, upon completion of CMOS processing $(a)$, beginning of anisotropic etch $(b)$, and after release of the micromachined circuit (c).

The resulting structure, shown in Figure l(c), contains a region of single crystal silicon suspended by dielectric support beams. Aluminum interconnects are embedded inside these oxide layers. Since the thermal conductivity of silicon dioxide and 
nitride is much lower than that of aluminum, the only significant thermal path through the supports is formed by these metal traces. This type of micromachined element provides circuitry with high thermal resistance to the substrate.

\section{IMPROVED TMAH ETCH CHEMISTRY}

The masking layer for the post-processing etch is the oxide or nitride passivation layer of the circuit process. The aluminum bond-pads are exposed during the etch. It is therefore necessary to use a silicon etchant with high oxide, nitride, and aluminum selectivity. Ethylene-diamene pyrocatechol (EDP) could be used as a silicon etchant, as certain formulations of this chemical have been shown to offer the required selectivity [5]. EDP however, is toxic and mutagenic. Similar safety concems, in addition to explosion hazards, apply to hydrazine silicon etchants. Tetramethyl ammonium hydroxide ('TMAH), another anisotropic etchant, also has very high oxide and nitride selectivity, and exhibits selectivity to aluminum if the solution is doped with silicon [6] or silicic acid $\left(\mathrm{Si}(\mathrm{OH})_{4}\right)$ [7]. TMAH is relatively safe to use, and presents no special disposal issues. The addition of silicates to the etchant acts in two ways to passivate the aluminum from attack by the alkaline etchant. The $\mathrm{pH}$ of the solution is reduced, but more importantly, the presence of silicates in solution will form aluminosilicates that are less soluble at moderate $\mathrm{pH}$ (between 8 and 12) than the hydrated aluminum oxides that would otherwise form at the metal surface. In the packaging industry, silicates are commonly used when aluminum is put into contact with alkali solutions of soaps or other cleaning agents [8]. The required amount of dissolved silicates is proportional to the concentration of the TMAH etching solution. In order to limit the amounts of silicon or silicic acid required in solution, a five weight percent TMAH solution was chosen. The passivation of aluminum requires at least $16 \mathrm{~g} / \mathrm{l}$ of dissolved silicon or $40 \mathrm{~g} / 1$ of dissolved silicic acid for this concentration.

One troublesome property of ТМAH is the formation of hillocks on (100) silicon for pH levels lower than 13 [9]. When these small pyramids form, the silicon etch rate drops dramatically. Figure 2 is a SEM photograph of a hillocked (100) p-type silicon surface. The mask was 100 . nm of thermal $\mathrm{SiO}_{2}$, and the etchant was $5 \mathrm{wt}$. \% TMAH with $16 \mathrm{~g} / 1$ of dissolved silicon at $80^{\circ} \mathrm{C}$.

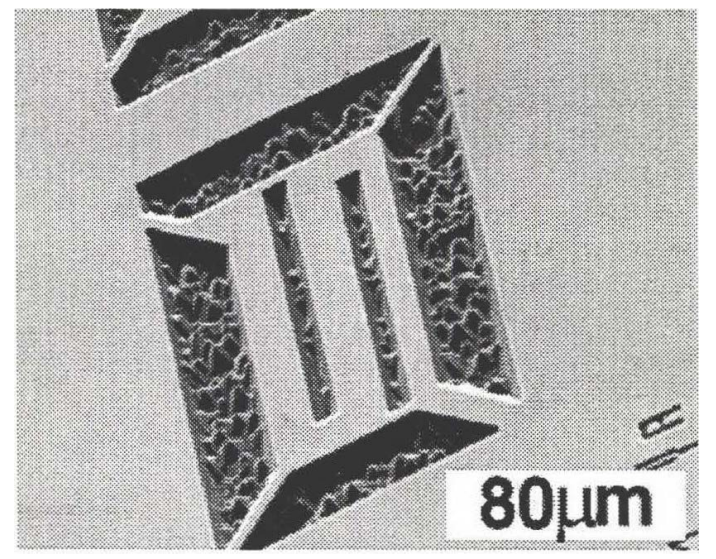

Figure 2. SEM photo of hillock formation on (100) silicon after etching in 5 wt. \% TMAH and $16 \mathrm{~g} / \mathrm{l}$ of dissolved silicon at $80^{\circ} \mathrm{C}$.

The alkaline silicon etching can be broken down into two separate steps and written as

$$
\begin{gathered}
\mathrm{Si}+2 \mathrm{OH}^{-} \rightarrow \mathrm{Si}(\mathrm{OH})_{2}^{2+}+4 e^{-} \\
\mathrm{Si}(\mathrm{OH})_{2}^{2+}+4 e^{-}+4 \mathrm{H}_{2} \mathrm{O} \rightarrow \mathrm{Si}(\mathrm{OH})_{6}^{2-}+2 \mathrm{H}_{2}
\end{gathered}
$$

In the first step, in Eqn. 1, electrons are injected into the conduction band of the silicon. These are used up in the reduction step in Eqn. 2. Hydroxyl ions from the water bind to the silicate formed in the first step, and produce soluble silicic acid, with hydrogen gas as a byproduct. Hillock formation is likely due to local micro-masking of the silicon. If small hydrogen gas bubbles cling to the surface before they release, they temporarily mask the surface undemeath from the etching solution. Hillocks form beneath these adhered bubbles. The bubbles block the free diffusion of reactants and products to and from the surface. A precipitate will form at the point of contact between the bubble and the silicon that creates a temporary masking layer as it is slowly dissolved upon release of the hydrogen bubble. This localized mask is composed of partially hydrated silicate species with variable stoichiometries. Initially the number of pyramids at the surface is small, but as etching continues new pyramids are formed and begin to superimpose over previous hillocks. Once the surface is fully covered with hillocks, the (100) etch rate will drop precipitously.

For increasing TMAH concentrations, the silicon etch rate decreases, and hillocking is reduced. This dependence on concentration, which occurs above two weight percent, is based on two key factors. First, as the concentration of water drops with increasing concentration, the formation of progressively less hydrated and less soluble silicate species reduces the rate of product removal from the surface. This effectively blocks the surface reduction reaction, until the products being solvated are removed. With further increases in concentration, the surface becomes more uniformly covered with these low solubility silicate species. The slightly soluble silicates that were located beneath bubbles for low concentration solutions are now spread unifonnly across the entire surface. The localized blocking effects are no longer important, and hillocking is reduced. The second factor is that the amount of water available for reduction by the silicon (Eqn. 2) is lower. This reduces the silicon etch rate, which in turn drops the formation rate and size of the bubbles clinging to the surface.

Palik, et al. [10] observed that when silicon is etched in $\mathrm{KOH}$, the average size of hydrogen bubbles diminishes with increasing $\mathrm{KOH}$ concentration, while the areal density of these bubbles goes up. They theorize that since smaller bubbles have a smaller "footprint" and release more quickly, they leave the surface less rough than larger bubbles with a longer "dwell time." The wetting properties of the solution may therefore in part determine the appearance of an etched surface. Surfactants could be added to TMAH to change the wetting angle of the solution on the silicon interface. This could keep bubbles from creating hillocks by reducing the dwell time. TMAH, however, is very sensitive to contamination from organic sources. The addition of a fluorochemical surfactant, FC-129 [11], to TMAH solutions caused silicon etching to stop. Ultrasonic agitation of the solution could also shorten the dwell time of adhered hydrogen. The agitation could, however, destroy micromachined devices. Another solution to the hillocking problem is therefore called for.

The addition of pyrazine to TMAH solutions has been shown to eliminate hillock formation [2]. This benefit did not apply to silicon-doped etching solutions, however. Certain potassium hydroxide $(\mathrm{KOH})$ - alcohol etchants also cause hillock formation. It has been reported that etching silicon in a $\mathrm{KOH}$ isopropanol solution saturated with oxygen eliminates hillock formation [12]. This method may yield similar benefits for TMAH 
solutions, but working with oxygen under high pressure presents an explosion hazard.

Alkaline silicon etches can produce smooth (100) surfaces if oxidizers are added to the etchant $[6,13]$. When an oxidizer with a higher redox potential than water is added to the etching solution, hydrogen gas evolution can be avoided. The oxidizer takes the place of water in Eqn. 2. If the byproducts of the solution are aqueous, no micro-masking by gas bubbles occurs and no hillocks form. This has been demonstrated with hydrogen peroxide in ammonium hydroxide, but the oxidizer concentration needs to be very tightly controlled [14]. If insufficient peroxide is added, hillock formation persists, but if too much is introduced, silicon etching ceases since the hydrogen peroxide rapidly reduces the $\mathrm{pH}$ of the etching solution. The required range was reported to be between $0.65 \times 10^{-2} \mathrm{M}$ and $1.84 \times 10^{-2} \mathrm{M}$.

Peroxydisulfates, another group of inorganic peroxides, are some of the strongest known oxidizers in aqueous solution [15]. This group includes potassium peroxydisulfate $\left(\mathrm{K}_{2} \mathrm{~S}_{2} \mathrm{O}_{8}\right)$ and ammonium peroxydisulfate $\left(\left(\mathrm{NH}_{4}\right)_{2} \mathrm{~S}_{2} \mathrm{O}_{8}\right)$. Peroxydisulfate is an even stronger oxidizer than peroxide. The half-cell reaction for the reduction of hydrogen peroxide can be written (relative to a standard hydrogen electrode, with $1 \mathrm{M}$ concentrations of each species) as

$$
\mathrm{H}_{2} \mathrm{O}_{2}+2 \mathrm{H}^{+}+2 e^{-} \leftrightarrow 2 \mathrm{H}_{2} \mathrm{O}
$$

and has a redox potential of $\mathrm{E}^{0}=1.776 \mathrm{~V}$ [16]. The redox reaction for the peroxydisulfate ion is

$$
\mathrm{S}_{2} \mathrm{O}_{8}^{2-}+2 e^{-} \leftrightarrow 2 \mathrm{SO}_{4}^{2-}
$$

which has a redox potential of $\mathrm{E}^{0}=2.01 \mathrm{~V}$.

Adding 5 to 10 grams per liter of either potassium or ammonium peroxydisulfate to TMAH solutions, even ones doped with silicon or silicic acid, eliminates hillock formation. In contrast to hydrogen peroxide, the exact peroxydisulfate concentration is not critical. The addition of.excess oxidizer does not stop, or even slow, the silicon etch rate.

Figure 3 shows the (100) silicon etch rate for a $5 \mathrm{wt}$. \% TMAH solution for several different temperatures. One set of data points represents the silicon etch rate with a pure solution, while the second shows the etch rate with the addition of 5 grams per liter of ammonium peroxydisulfate. The surface of the silicon etched in the pure TMAH-water solution was hillocked, while that for the solution with added oxidizer was smooth. The data was taken for 5-10 $\Omega \cdot \mathrm{cm}$ p-type silicon, using a $100 \mathrm{~nm}$ thermal oxide mask.

Figure 4 shows SEM photos of two sets of elements used in a thermal RMS converter [17]. The devices in Figure 4(a) were etched in a $5 \mathrm{wt}$. \% TMAH solution containing $16 \mathrm{~g} /$ of dissolved silicon. While the aluminum bond-pads were not attacked by the etchant, there is significant hillocking at the bottom of the cavity. The devices are thermally isolated from the substrate, but the cavity is only 45-50 $\mu \mathrm{m}$ deep. The photo in Figure 4(b), however, shows an identical set of thermo-elements etched in a solution containing $5 \mathrm{~g} / \mathrm{l}$ of ammonium peroxydisulfate. The cavities under these devices have smooth bottoms (not visible) and are approximately $125 \mu \mathrm{m}$ deep. The etch time for each of these sets of devices was approximately 150 minutes at $80^{\circ} \mathrm{C}$.

Silicon doped TMAH solutions, with or without added oxidizers, have been found to effectively etch silicon for about one day. The $\mathrm{pH}$ of the solution drops to unacceptably low levels after 6 to 8 hours. The time will be shorter if large volumes of silicon are etched during use. This effect could be mitigated by appropriately buffering the $\mathrm{pH}$ level of the solution. Even without this added measure, however, the low cost of the solution and ease of disposal make it feasible to use a fresh solution for each day of use.

Etch Rate $(\mu \mathrm{m} / \mathrm{min})$

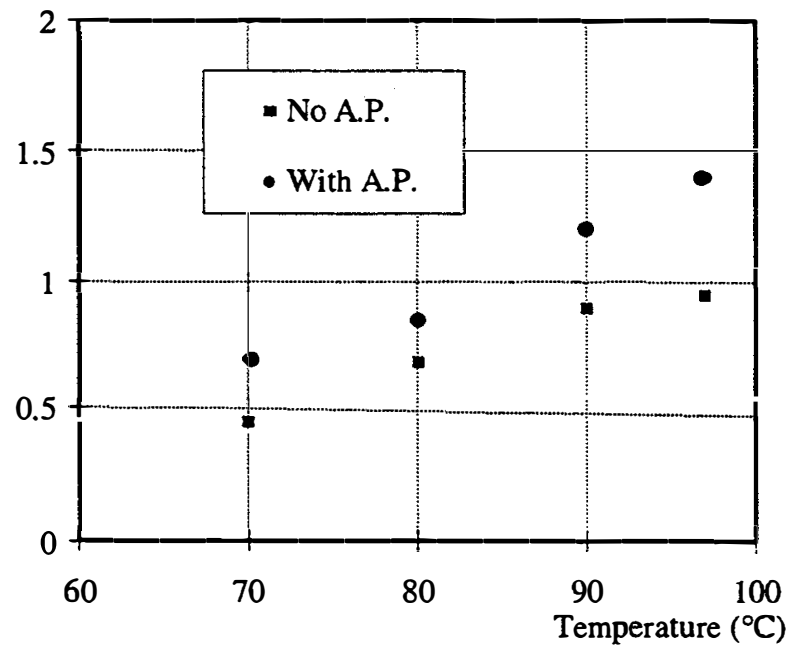

Figure 3. (100) silicon etch rate at several temperatures for $5 \mathrm{wt}$. $\%$ TMAH with and without added ammonium peroxydisulfate. No dissolved silicates were added to these solutions.

\section{AUTO-BIASED ETCHING}

Since the etch is a post-process step, the circuitry built during the CMOS process is functional during etching. Portions of these circuits can play an active role during the micromachining etch step. One application for this is the distribution of the passivation potential to multiple n-wells. Figure 5 shows a device configuration that achieves this. In this case, each element contains a suspended diode, which are series connected, as shown. Many other configuration are of course also possible. Each of the n-wells must be maintained at the passivation potential during the undercutting etch. To prevent having to short-circuit the wells together permanently, a series of transmission gates are used. The passivation potential is applied to the lower signal line, labeled $\mathrm{V}_{\mathrm{pp}}$. In solution this represents a voltage of $-0.8 \mathrm{~V}$ relative to the reference electrode. A control signal, $\mathrm{V}_{\mathrm{etch}}$ is then applied to turn the NMOS transmission gates on. This voltage must be more than one threshold voltage greater than the passivation potential. With the transmission gates tumed on, each n-well shown is electrically connected to the passivation potential. Following the etch, the transmission gates can simply be turned off by keeping the two lines at the lowest potential on the chip $\left(\mathrm{V}_{\mathrm{ss}}\right)$. The $\mathrm{n}$-wells are then electrically disconnected from each other.

This concept is demonstrated by the 70 element array of test devices shown in Figure 6 . The transmission gates are located between each row of elements. The passivation potential was introduced at a single point, and distributed by the transmission gates to each individual element. 


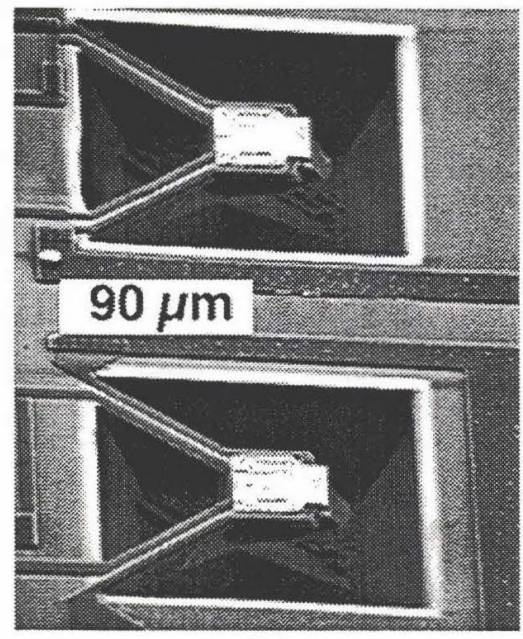

(a)

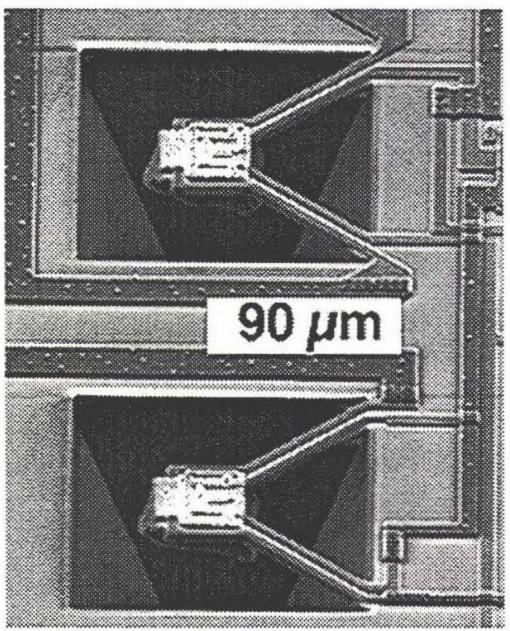

(b)

Figure 4. Two SEM photos of thermal RMS converter elements. The cavities under the devices in (a) are covered with hillocks, while those in (b) are smooth, due to the addition of peroxydisulfates to the silicon-doped TMAH solution.

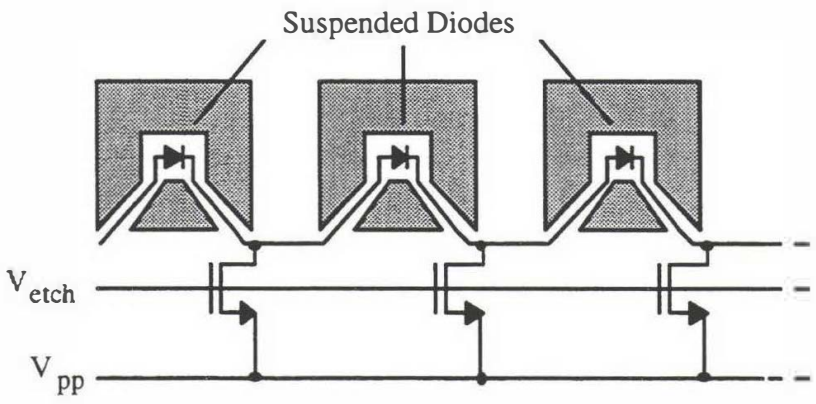

Figure 5. Schematic of a configuration that employs on-chip circuitry during the micromachining etch step.

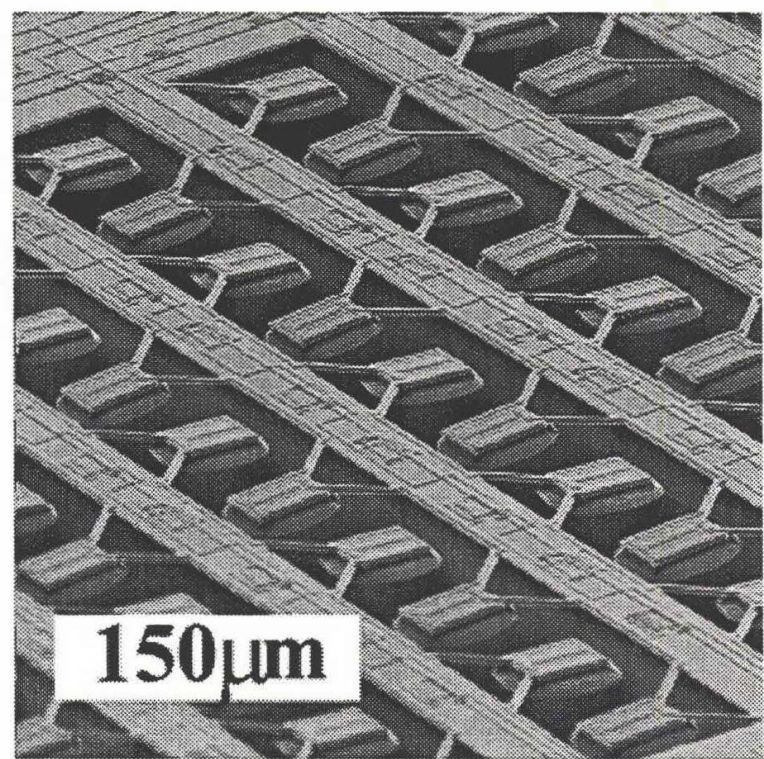

Figure 6. An array of 70 suspended n-wells fabricated using auto-biased etching.

\section{APPLICATIONS AND RESULTS}

The silicon undercutting technique has been employed on the Mosis $2.0 \mu \mathrm{m}$ Analog CMOS process, as well as on an Analog Devices BiCMOS process. Thermal isolation up to $50,000 \mathrm{~K} / \mathrm{W}$ has been achieved.

The technology presented here has been used to fabricate a thermal AC to RMS converter with a $60 \mathrm{~dB}$ dynamic range. Figure 4 is an SEM of a pair of thermoelements used in this RMS converter. Each of the matched elements contain a polysilicon resistor and a diode [17]. The resistor is used as a heater, while the diode is used under forward-bias as a temperature sensor. One of the two elements is heated by the AC signal. The second element is heated with a DC signal by feedback circuitry, such that the two elements are maintained at equal temperatures. The DC signal is the RMS value of the AC signal. The control circuits for the RMS converter system were integrated on-chip, alongside the micromachined elements. The converter has a bandwidth in excess of $400 \mathrm{MHz}$ and a nonlinearity better than $1 \%$.

Another application for this thermal isolation method is temperature-regulation of analog circuits. This technique can be used to mitigate the effects of ambient temperature changes on precision circuitry, such as voltage references. A bandgap voltage reference that employs such temperature regulation was built [18]. Figure 7 is an SEM of such a suspended circuit. The device contains PMOS heating transistors, which, together with a feedback network, maintain the silicon underneath the rectangular element at a constant temperature. The output voltage drift of the device was reduced by a factor of 40 , to $9 \mathrm{ppm} /{ }^{\circ} \mathrm{C}$, by using temperature regulation. The high thermal isolation of the circuits enabled a power dissipation of only $2 \mathrm{~mW}$. Work is currently underway to further improve the performance of a micromachined voltage reference. 


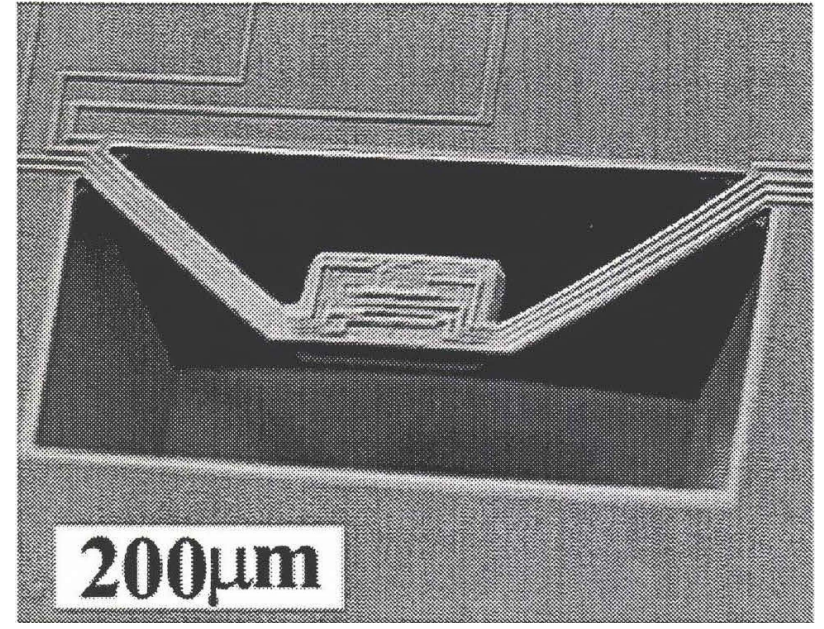

Figure 7. SEM of a micromachined bandgap voltage reference.

\section{CONCLUSIONS AND OUTLOOK}

We have presented a new IC post-processing method that requires no additional masking steps beyond those used for the circuit fabrication. The technique is capable of providing circuitry with thermal isolation as high as $50,000 \mathrm{~K} / \mathrm{W}$ at atmospheric pressure. An improved TMAH etching chemistry uses strong oxidizers to eliminate hillock formation. The resulting etchant provides good silicon etch rates, and exhibits high selectivity to oxide, nitride, and aluminum. Since the undercutting etch is performed as a post-process step, finished circuitry from the CMOS process can play an active role during the micromachining operation. A method to use transmission gates for the distribution of the electrochemical bias was described. In this fashion, many separate islands of suspended silicon can be realized. Two applications of the technology, an RMS converter and a temperature-regulated circuit, were discussed as examples. The micromachined bandgap voltage reference demonstrates a use for micromachining in improving the performance of integrated circuits.

To date, the process has been sucessfully implemented using two different IC technologies: a $2.0 \mu \mathrm{m}$ n-well analog CMOS process offered through MOSIS, and an Analog Devices BiCMOS line.

\section{REFERENCES}

1. R. Lenggenhager, H. Baltes, J. Peer, and M. Forster, "Thermoelectric infrared sensors by CMOS technology," IEEE Electron Device Letters, No. 13, pp. 454-456, 1992.

2. R. Lenggenhager, D. Jaeggi, P. Malcovati, H. Duran, H. Baltes, E. Doering, "CMOS membrane infrared sensors and improved TMAHW etchant," In Tech. Dig., IEEE Intl Elect. Dev. Meeting, San Francisco, CA, Dec. 11-14, 1994, pp. 531-534.

3. M. Parameswaran, H. Baltes, L. Ristic, A. Dhaded, and A. Robinson, "A new approach for the fabrication of micromechanical structures," Sensors and Actuators, vol. 19, pp. 289-307, 1989.

4. B. Kloeck, S. D. Collins, N. F. de Rooij, and R. L. Smith, "Study of electrochemical etch-stop for high-precision thickness control of silicon membranes," IEEE Trans. Electron Devices, vol. 36, no. 4, pp. 663-669, 1989.

5. D. Moser, "CMOS flow sensors," Ph.D. Dissertation, Physical Electronics Laboratory, ETH Zurich, p. 96.

6. U. Schnakenberg, W. Benecke, P. Lange, "TMAHW etchants for silicon micromachining," In Proceedings, Transducers '91, San Francisco, CA, pp. 815-818, 1991.

7. G. Lin, B. Eyre, K. Pister, "Bulk micromachining of standard CMOS using silicic acid added to TMAH for aluminum passivation," UCLA MEMS Laboratory internal report, TRN 95-13, June 1995.

8. The Corrosion Handbook, John Wiley, New York, p. 915, 1948

9. O. Tabata, R. Asahi, H. Funabashi, S. Sugiyama, "Anisotropic etching of silicon in $\left(\mathrm{CH}_{3}\right)_{4} \mathrm{NOH}$ solutions", In Proceedings, Transducers '91, San Francisco, CA, pp. 811-814, 1991.

10 . E.D. Palik, O.J. Glembocki, I. Heard, P.S. Bumo, L. Tenerz, "Etching roughness for (100) silicon surfaces in aqueous $\mathrm{KOH}, " \mathrm{~J}$. Appl. Phys., vol. 70, no. 6, pp. 3291-3300, 1991.

11. $3 \mathrm{M}$ Fluorad Fluorochemical Surfactants, $3 \mathrm{M}$ Specialty Chemicals Division, 908 North Elm St., Hinsdale, IL 60521.

12. S.A. Campbell, K. Cooper, L. Dixon, R. Earwaker, S.N. Port, and D.J. Schiffrin, "Inhibition of pyramid formation in the etching of $\mathrm{Si}$ p $<100>$ in aqueous potassium hydroxide-isopropanol" J. Micromech. Microeng. nol. 5, pp. 209-218, 1995.

13 . W. C. Erdman, P. F. Schmidt, "Anisotropic etching of silicon and germanium," US Pat No. 3,738,881, June 12, 1973.

14. U. Schnakenberg, W. Benecke, B. Loechel, S. Ullerich, and P. Lange, " $\mathrm{NH}_{4} \mathrm{OH}$ based etchants for silicon micromachining: influence of additives and stability of passivation layers," Sensors and Actuators, vol. A25-27, pp. 1-7, 1991.

15. Kirk-Othmer Encyclopedia of Chemical Technology, Third Edition, John Wiley \& Sons, New York, volume 17, pp. 15-19.

16 Handbook of Chemistry and Physics, 66th edition, CRC Press, 1986, p. D157.

17. E. H. Klaassen, R. J. Reay, G.T.A. Kovacs, "Diode-based thermal RMS converter with on-chip circuitry fabricated in CMOS," In Transducers '95 Tech. Dig., Stockholm, pp. 154-157, 1995.

18 . R.J. Reay, E.H. Klaassen, G.T.A. Kovacs, "A low-power temperature-regulated bandgap voltage reference," In Tech. Dig., ISSCC '95, San Francisco, pp. 166-167, 1995. 